\title{
Für eine milieusensible Kommunikationsstrategie
}

Auf der Grundlage einer differenzierten Beschreibung und Analyse gesellschaftlicher Teilgruppen bieten die zugänglichen Sinus-Milieustudien (vgl. Barz/Tippelt 2004; Wippermann/Magalhaes 2005) einen reichhaltigen Zugewinn an Wissen über die sozialen Milieus sowie bislang weitgehend ausgeblendete Bezüge, nämlich über die milieuspezifischen Grundannahmen, die damit verbundenen religiösen und weltanschaulichen Perspektiven und über die kirchlich-kommunikativen Anschlusschancen. Trotz einiger durch sie aufgeworfenen theoretischen und methodischen Anfragen (vgl. Ebertz 2006) werfen die Studien zugleich einen erheblichen Zugewinn an Einsichten dahingehend ab, die Resonanzfähigkeit medialer Produkte (und pastoraler Angebote) im Blick auf die Rezeptionsmuster unterschiedlicher sozialer Milieus zu steigern. Die Befunde der Sinus-Milieustudien basieren auf der Dauerbeobachtung soziokultureller Milieus und finden deshalb, aber auch wegen ihres vergleichsweise „detailgenaueren Blicks“ (Müller-Schneider 2003) auf eine Wirklichkeit, die in Deutschland seit den 1990er Jahren trotz gewisser Variationen und Innovationen von Lebensstilen und Milieus eine äußerst stabile Grundarchitektur im Milieugefüge aufweist, hohe Aufmerksamkeit in der Soziologie. Tatsächlich steckt in ihnen auch eine Vielfalt an Chancen, den kommunikativen Horizont der Kirche zu erweitern und diese selbst kommunikativ zu bereichern.

Ob sich die Einsichten der Sinus-Forschung auf der praxeologischen Ebene im pastoralen Bereich im allgemeinen und im medialen Bereich im besonderen lohnen und bewähren, ist freilich eine andere Frage. Der wirkliche Erfolg einer differentiellen Milieubetrachtung hängt weniger von den vorliegenden Sinus-Studien ab, als davon, ob seitens der kirchlichen Träger der pastoralen und medialen Kommunikation der Frohen Botschaft tatsächlich Bereitschaft besteht, solche Ergebnisse ernst zu nehmen und sich auf sie einzulassen. Angesichts bestimmter, teilweise wie mir scheint - nicht gerade durch Klugheit und Intelligenz geleiteter Reaktionen, welche die Befunde der Milieustudie indirekt bestätigen, ist hier allerdings ein dickes Fragezeichen zu machen. Es wird keinen Konsens hinsichtlich des Nutzens der Sinus-Milieustudien geben; die Interessen, die mit ihr verbunden sind, und die Kirchenbilder der Rezeptoren sind maximal divergent. Wenn die Sinus-Milieuforschung nach einer Phase der Aufgeregtheiten gleichwohl handlungsleitend werden sollte, dann steht nicht nur die Pastoral überhaupt, sondern auch die ihr dienende mediale kirchliche Kommunikation vor Aufgaben von bislang nicht 
gekannten Ausmaßen. Dies führt mich zu den nachfolgenden - allerersten - praxeologischen Vorschlägen (unter der Akzentuierung des medialen Bereichs).

Die Verwertung der durch die vorliegenden Sinus-Studien zu gewinnenden Erkenntnisse über milieuspezifische Religions- und Kirchenverhältnisse bzw. über milieuspezifische pastorale und kirchenmediale Anschlusschancen legt Reflexions- und Implementationsstrategien nahe, welche die mittel- und langfristige Kommunikationsplanung einschließlich der Unterstützungsmaßnehmen zu deren Umsetzung betreffen. Solche Reflexions- und Implementationsstrategien wären als eigenständige Beratungsprojekte in Kooperation mit den unterschiedlichen Trägern der medialen / pastoralen Kommunikation der Kirche detailliert zu erarbeiten und zu evaluieren. Hierzu kann das kommentierte Zielgruppen-Handbuch von Sinus Sociovision, aber auch die Studie von Barz und Tippelt außerordentlich wichtige Hinweise geben.

\section{Sehen}

Einer der ersten Schritte einer solchen Reflexions- und Implementationsstrategie ist aus meiner Sicht die Intensivierung der Wahrnehmung des Bestehenden: die Wahrnehmung und Vertiefung der Wahrnehmung, dass es solche Milieus überhaupt gibt, welche die Lebensführung und die basalen Orientierungen und das Zusammenleben bestimmen, was solche Milieus sind und was nicht. Dabei geht es auch um den Abbau von nicht $z \mathfrak{u}$ unterschätzenden Widerständen, solchen Milieu-Formationen gewissermaßen die Anerkennung der Wahrnehmung zu verleihen. Blindheitsverabredungen sind in der Kirche in vielerlei Hinsicht gängig und gerade auch im Blick auf die Reaktionen auf die ,katholische' Sinus-MilieuStudie erkennbar, nach dem Motto: Der Kirche gehe es doch

- um den Menschen, nicht um Milieus, also um anthropologische Konstanten (als gäbe es diese konkret jenseits der jeweiligen soziohistorischen Sozialnatur; als könne man mit einem Kind genauso kommunizieren wie mit einem Erwachsenen, mit einem Deutschen genauso wie mit einem Italiener, mit einem Asiaten ähnlich wie mit einem Europäer usw.);

- um die individuelle Person, die in kein (Milieu-)Schema passe (als hätte es noch nie eine soziokulturelle Differenzierung in der Pastoral und massenmedialen Kommunikation der Kirche gegeben);

- um eigene Milieubildungen, nicht um Anpassung an fremde Milieus (als könnte die Kirche die sozialmoralischen Konfessionsmilieus des 19. Jahrhunderts wieder restituieren); 
- um überhaupt keine Milieubildung, weil dies ins Getto führe (als wäre das katholische Milieu der Vergangenheit nur Getto gewesen);

- um das, was alle Milieus miteinander - transversal - verbinde, was sie unterfange oder überwölbe (als könnte man in einer differenzierten Gesellschaft noch diese Integrationsleistung vollbringen).

Eine Wahrnehmungsschulung steht an, z. B.:

- bezogen auf die Milieulandschaft: Welche milieugeprägten Orte und Dinge gibt es im öffentlichen Raum (z. B. Häuser, Automarken, Läden, Gasthäuser, Kleidung, Körperpräsentationen)?

- bezogen auf die Kommunikatoren selbst: Welchem Milieu gehören sie - als Kommunikatoren - an? Welche Präferenzen haben sie, welche Distinktionen, welche Abwertungen usw.? Wie ist ihre potentielle Milieureichweite, d.h. welche Milieus könnten sie erreichen, welche nicht?

- bezogen auf die eigenen Medien-Produkte: Welches Milieu bedienen sie (faktisch), welches ignorieren sie (ungewollt) usw.? In welchen Milieus sind sie stark, schwächer oder gar nicht präsent? Wie lassen sich die kirchlichen Medienprodukte nach ihren (vielleicht unterschwelligen) Milieupräferenzen sortieren und gewichten?

Ziel dieser Wahrnehmungsschulung ist die Überprüfung der bisherigen medialen/publizistischen Formate auf die stillschweigenden Selektionen und Milieuverengungen sowie die Erkenntnis der - vermutlich einseitigen, beschränkten - Reichweite eines Großteils der medialen kirchlichen Produkte, wenn nicht des Spektrums der medialen kirchlichen Produkte überhaupt.

Für jedes der zehn von Sinus Sociovision konstruierten Milieus verfügt die vorliegende ,katholische‘ Sinus-Studie über ein klares Porträt, das wie eine Folie an die eigenen Kommunikationsprodukte herangetragen werden kann. Milieu für Milieu werden die neu erhobenen Bausteine zum „Lebenssinn“, zur „Weltanschauung“, zu „Religion und Kirche“, zur „Nutzung und Bedeutung der Bibel“, zum jeweiligen „Image der katholischen Kirche“, zu den „Wünschen und Forderungen an die Kirche“ zusammengetragen. Wer die Befunde aus Barz/Tippelt (2004) hinzulegt, kann die Milieuporträts weiter unterscheiden nach prägenden Bildungserfahrungen in Kindheit und Jugend, den Bildungsvorstellungen, den Weiterbildungsinteressen und -barrieren, dem Persönlichkeitsverständnis, dem Gesundheitsbewusstsein und -verhalten sowie nach den typischen Ansprüchen an die kommunikativen Methoden und den ästhetischen Stil.

Mit Hilfe der Sinus-Milieustudie gehen einem in der Tat die Augen auf, und man beginnt zu verstehen, weshalb dieses oder jenes (kirchliche) Angebot bei diesen oder jenen auf Nachfrage stößt und anderes nicht. 
Und man beginnt zu fragen, ob und inwieweit in kirchlichen Einrichtungen und Organisationen Milieubarrieren wirksam sind, die dadurch selektiv wirken und auch Ressentiments hervorrufen, dass ganz bestimmte Milieumerkmale prämiert werden; dass die kirchlichen Gelder asymmetrisch auf die Milieus verteilt sind. Vielleicht wird man auch erkennen, dass als persönlich eingeschätzten Kommunikationsproblemen häufig Milieukonflikte zu Grunde liegen.

\section{Urteilen}

Ein weiterer Schritt müsste darin bestehen, die selbstverständlichen Grundannahmen, welche Aufgaben mit der medialen Kommunikation der Kirche erreicht werden sollen, bewusst zu machen. Es müsste darum gehen, solche fraglosen Einstellungen mit den Ergebnissen der MilieuStudie zu konfrontieren, die ja eine ausgesprochen differentielle Kommunikation nahe legt. Sinus Sociovision geht zwar einerseits davon aus, dass „die Grenzen zwischen den Milieus fließend sind“, betont jedoch auch, dass „ein wirkliches wechselseitiges Verstehen zwischen Menschen aus verschiedenen Milieus nicht oder nur begrenzt möglich" ist (Wippermann/Magalhaes 2005, S. 7). Wie vertragen sich solche Befunde z. B. mit der binnenkirchlich so beliebten Rede von ,dem Menschen', mit den communialen Kirchenbildern, mit der Unterstellung milieuübergreifender oder milieuunterfangender - milieutransversaler - Kommunikationsmöglichkeiten?

Hilfreich könnte in diesem Zusammenhang auch der Hinweis sein, dass die Kirchengeschichte 2. B. des 19. Jahrhunderts ein weitaus höheres Differenzierungsniveau kannte als die Kirchengeschichte der letzten 50 Jahre. Überprüft werden müssten die medialen Kirchenprodukte auch auf ihren Erfolg hin: Welchen Erfolg haben milieuignorante, welchen Erfolg haben - möglicherweise ja bereits vorhandene - milieuspezifische Produkte? Welche kirchenverwandten Beispiele können (etwa durch Sinus) empfohlen werden, an denen eine Steigerung des Kommunikationserfolgs über die Orientierung an den Sinus-Milieus nachweisbar ist, um als ermutigend $z \mathfrak{u}$ wirken?

Ziele dieser Beurteilung sind die Überprüfung der Verträglichkeit der Milieuphilosophie mit der Kirchenphilosophie und der Abbau von Widerständen, in den Milieukategorien zu denken, sowie zu lernen, sich mit Hilfe der Milieukategorien die kommunikative Welt neu zu erschließen. 


\section{Handeln}

Hier müsste die Frage im Zentrum stehen, wie die kirchen-mediale Landschaft umzugestalten wäre, wenn sie sich auf die Milieubefunde einließe. Kann es dann beispielsweise noch so etwas wie Bistumszeitungen geben? Welche Milieu-Lücken sind zwischen „Rheinischer Merkur“, „Christ in der Gegenwart“ und „Publik Forum“ zu schließen? Die Liste der mittel- und langfristigen Aufgaben reicht bis hinein in personalstrukturelle Überlegungen für eine neue, am differentiellen Milieumarketing ausgerichtete Medienoffensive der Kirche bzw. mit ihr verbundener selbständiger - Verlage.

Ziele dieser Handlungsstrategie sind die Erschließung neuer MilieuAdressaten, die bislang kirchenkommunikativ vernachlässigt wurden, die Verwandlung überkommener kirchlicher Medienprodukte und der Aufbau neuer Medienprodukte in Richtung einer stärkeren Milieudifferenzierung und -spezialisierung sowie eine stärkere Konzentrierung und Konzertierung der kirchlichen Kommunikatoren im Blick auf die unterschiedlichen Milieuformationen.

Wenn es zwischen den Milieus erhebliche Kommunikationsschwellen gibt und somit die Unwahrscheinlichkeit wächst, alle Milieus über ein Kommunikationsmedium - etwa das Pfarrblatt oder die Bistumszeitung erreichen $z$ k können, läge eine entscheidende kirchenpublizistische Herausforderung darin, neue Gelegenheiten der Kommunikation (der Frohen Botschaft) $z \mathfrak{u}$ erschließen und diese komplexer $z \mathfrak{u}$ gestalten. Kommunikative Anpassung scheint angesagt. Der hier bewusst gewählte Begriff der Anpassung, den die Texte des Zweiten Vatikanums mehr als fünfzigmal verwenden (zwar in semantischen Varianten, aber durchgehend positiv), meint ja - gerade in der stets $\mathrm{zu}$ betonenden Unterscheidung zum Begriff der Assimilation - nicht Konformismus, Außenlenkung, Opportunismus, Anbiederung usw. Dies ist auch mit dem theologischen Begriff der Anpassung des Zweiten Vatikanums (und des Kirchenrechts!) nicht gemeint. Von einer solchen schwachen oder passiven Anpassung, wozu übrigens einige kirchliche Bereiche gleichwohl neigen, ist die aktive Anpassung $z u$ unterscheiden. Diese sucht kommunikative Anschlussfähigkeit, ohne ihr Proprium preiszugeben; sucht kommunikative Gestaltung, um vorgefundene Lebenswelten und Lebensformen (= Milieus) auch $z \mathfrak{u}$ interpretieren, neu- und umzuinterpretieren, $z \mathfrak{u}$ verändern und neu zu gestalten, freilich immer nur als einladendes Angebot und als Chance zum eigenen Lernen und Umlernen.

Was das Dekret des Zweiten Vatikanums über die Missionstätigkeit der Kirche (Ad Gentes 22) bei den „verschiedenen Völkerfamilien“ sagt, könnte auch auf die innere Mission bei den verschiedenen Milieustäm- 
men moderner Gesellschaften bezogen werden: „Wenn man so vorangeht, wird jeder Anschein von Synkretismus und falschem Partikularismus ausgeschlossen; das christliche Leben wird dem Geist ủnd der Eigenart einer jeden Kultur angepasst; die besonderen Traditionen, zusammen mit den vom Evangelium erleuchteten Gaben der verschiedenen Völkerfamilien, werden in die katholische Einheit hineingenommen. So haben schließlich die jungen Teilkirchen mit dem ganzen Reichtum ihrer Überlieferung ihren Platz in der kirchlichen Gemeinschaft, unter voller Wahrung des Primats des Stuhles Petri, der in der ganzen Gemeinschaft der Liebe den Vorsitz führt." Wer das „missionarisch Kirche sein in der Welt von heute" ernst nimmt, könnte mit Hilfe der neuen Sinus-Studie ernst machen. Die Sinus-Milieus sind nämlich so etwas wie innergesellschaftliche Kulturen, allerdings keine festen Gruppen oder Systeme. Über den Aggregatzustand von Milieus ist deshalb abschließend noch zu reflektieren, zumal dies von hoher kommunikationsstrategischer Relevanz sein dürfte.

\section{Nochmals sehen: Was Milieus sind und was sie nicht sind}

Der Ausdruck Milieu, wie er in den Sinus-Studien Verwendung findet, ist zwar ein Kollektivbegriff, er meint aber trotz manch missverständlicher Aussagen zunächst keinerlei Interaktions-, Gruppen-, Gemeinschaftsoder Organisationsgeschehen (wie Gespräch im Zugabteil, Familie, Verein, Verband) und zielt somit auch auf keinen kollektiven Akteur. Ähnlich wie die Begriffe Klassenlage oder die Generationslage meint er einen bloßen Zusammenhang: ein Miteinander und eine Verbundenheit also ohne Zusammenleben oder Zusammenschluss. Das Etwas, durch das man im Milieu verbunden ist, lässt sich mit einem Ausdruck Karl Mannheims zunächst als „Lagerung“ (Mannheim 1964, S. 525) bezeichnen.

\section{Milieulagerung}

Wie man „unter Klassenlage [...] eine schicksalsmäßig verwandte Lagerung bestimmter Individuen im ökonomisch-machtmäßigen Gefüge der jeweiligen Gesellschaft“ verstehen kann und die Generationslage „auf einer verwandten Lagerung der einer Generation zurechenbaren Individuen im sozialen Raume beruht" (Mannheim 1964, S. 525f.), sind auch die Sinus-Milieus als Lagerungen von einander materiell wie immateriell und auch generationell einander ähnlichen Individuen begreifbar. Eine Lagerung muss nicht damit einhergehen, dass die Zugehörigen um sie wissen, sich ihr bewusst zurechnen, gar eine Klassen-, Generations- oder 
Milieu-Identität haben, auf die sie stolz sind. Ähnlich wie die Klassenlage nur im individuellen oder kollektiven Auf- oder Abstieg verlassen werden kann, besteht auch eine prinzipielle Inter-Milieu-Mobilität, die allerdings schon durch den Zusammenhang mit der Generationslage, die ein Milieu mitbestimmt, begrenzt ist. Einem Aufstieg etwa aus den Sinus-Milieu der Hedonisten oder der Konsum-Materialisten zum Milieu der Konservativen dürften von daher natürliche Grenzen gesetzt sein. Ein Gemeinsames von Klassenlage, Generationslage oder Milieu ist deshalb eher darin zu sehen, dass sie Denk-, Erlebens- und Handlungsspielraum begrenzt: „Eine jede Lagerung schaltet also primär eine große Zahl der möglichen Arten und Weisen des Erlebens, Denkens, Fühlens und Handelns überhaupt aus und beschränkt den Spielraum des sich Auswirkens der Individualität auf bestimmte umgrenzte Möglichkeiten." (Mannheim 1964, S. 528)

Zugleich zeigt das Milieu wie jede Lagerung „im positiven Sinne eine Tendenz auf bestimmte Verhaltungs-, Gefühls- und Denkweisen, die aus dem eigenen Schwergewicht der Lagerung heraus [...] verstehend erfassbar ist" (Mannheim 1964, S. 528). Dementsprechend haben die Milieus, wie die katholische Sinus-Studie zeigt, z. B. unterschiedliche Lebenskonzepte, Sinngebungen, Lebensträume und auch Kirchenbilder. Kirche ist gewissermaßen für jedes Milieu nur in einem bestimmten Aspekt oder in bestimmten Hinsichten vorhanden, während ihm andere Perspektiven verschlossen bleiben. Es gibt milieuspezifische Sichtweisen auf und Zugangsweisen zur Kirche, und auch die Art und Weise der Hinwendung zu ihr und der Verarbeitung ihrer Traditionen und Angebote werden verstehbar aus der jeweiligen Milieu-Lagerung, die auch mit einer spezifischen sozialen Lagerung (Einkommens-, Berufs- und Bildungs-Schicht) und Generationslagerung (A, B, C) einhergeht. Wenn es stimmt, dass „nacheinander folgende Generationen stets einen jeweils anderen Gegner in der Welt und in sich bekämpfen“, wie Karl Mannheim (1964, S. 537) sagt, dann sind die jeweils einem Sinus-Milieu Zugehörigen nicht nur mitformatiert durch in ihrer Jugend erhaltene Ersteindrïcke, sondern auch durch jene von ihm auch so genannte „Polarerlebnisse". Und in solchen Tiefenschichten unterscheiden sich eben auch die jeweiligen Milieus, was sie auch einander fremd macht.

Eine weitere Differenzierung des Milieu-Begriffs ist zu klären, wenn wir noch die Unterscheidung von Milieuzusammenhang und Milieueinheit vornehmen. Dann zeigt sich deutlicher: Während innerhalb der oder zumindest einiger - Milieus konjunktive Erfahrungen gegeben sind, die in einem gemeinsamen Erleben verankert sind, sind zwischen - zumindest den meisten von - ihnen allenfalls kommunikative Erfahrungen möglich; d.h. Intersubjektivität zwischen den Vertretern von Milieus 
muss immer erst hergestellt werden und bleibt deshalb prekär (vgl. Bohnsack 1993, S. 64). Gerade für kommunikationsstrategische Planungen ist dies $\mathrm{zu}$ berücksichtigen.

\section{Milieuzusammenhang, Milieueinheit und Milieugruppe}

Mit Milieuzusammenhang ist noch mehr als die bloße Präsenz in einer bestimmten sozialen Einheit gemeint, die durch Beruf, Bildung und Einkommen sowie Generationslagerung und Grundwertorientierung bestimmt ist. Wie Karl Mannheim das Mehr eines Generationszusammenhangs gegenüber der bloßen Generationslagerung darin sieht, dass gleichaltrige Individuen tatsächlich ,an jenen sozialen und geistigen Strömungen teilhaben, die [...] den betreffenden historischen Augenblick konstituieren" (Mannheim 1964, S. 543), also Gemeinsamkeiten des historischen Schicksals haben, müsste auch im Blick auf soziale Milieus eine Unterscheidung getroffen werden können. Ähnliches gilt auch hinsichtlich des Begriffs der Generationseinheit, die - wie die so genannte 68er-Generation - im „Kern durch eine konkrete Gruppe gebildet wird“ (Mannheim 1964, S. 548) und darin auch historische Bezugspersonen und Bezugsgruppen hat, ohne in einem solchen kollektiven Akteur aufzugehen. Tatsächlich scheint das eine oder andere Sinus-Milieu - so das Nachkriegs-Milieu der Traditionsverwurzelten oder das Milieu der Hedonisten - eher den Charakter eines durch eine gemeinsame Sozialisationsgeschichte und ein gemeinsames biographisches Erlebens geprägten Milieuzusammenhangs, ja sogar - wie z. B. das Alt-68er-Milieu der Postmateriellen und das Milieu der DDR-Nostalgiker - einer Milieueinheit zu tragen, ohne dass dies für alle Sinus-Milieus zuträfe. Mit anderen Worten: Den Sinus-Milieus liegen nicht nur unterschiedliche, sondern teilweise auch andersartige existentielle und erlebnismäßige Verbundenheiten zu Grunde.

So ist auch fraglich, ob sich hinter den zehn so genannten ,gesamtdeutschen Milieus' von Sinus-Sociovision nicht auch unterschiedliche Milieuzusammenhänge oder Milieueinheiten verbergen, die bei einer differentiellen Milieu-Analyse zum Vorschein kämen. Das Milieu z. B. der Traditionsverwurzelten in der ehemaligen DDR unterscheidet sich als Milieuzusammenhang ganz gewiss von demjenigen der Traditionsverwurzelten in der ehemaligen Bundesrepublik Deutschland, wird aber in den aktuellen Sinus-Studien - wie auch in der vorliegenden - ungeschieden präsentiert. So hat sich bei den Katholiken und Katholikinnen Ostdeutschlands eher die Vorstellung einer positiven Zuordnung von Kirche und Freiheit entwickelt als in Westdeutschland, die auf einer andersartigen existentiellen oder erlebnismäßigen Verankerung beruht und 
räumlich zerstreute Individuen einer konfessionellen Minderheit zu einer Einheit verbunden hat. Diese „konjunktiven Erfahrungen“ (Mannheim 1980, S. 285f.) dürften sich auch auf gemeinsame (pastorale) Gestaltungsprinzipien bezogen haben, die man für ebenfalls konstitutiv halten kann für die Formierung sozialer Milieueinheiten. Bleibt noch anzumerken, dass die vorliegende ,katholische' Sinus-Studie zeigt, dass sich Milieus in unterschiedlichen Milieugruppen vergesellschaften - gleichsam als spezifische, durch direkte kommunikative Beziehungen bestimmte Ausprägungen konjunktiver Erfahrungsräume der sozialen Großmilieus. Während sich die generationell älteren Milieus (aus dem Sinus-A- und B-Bereich) z. B. in Vereinen und Clubs vergesellen und darunter die statushohen Milieus eine deutliche Neigung nicht nur zur aktiven sozialen Schließung, sondern auch zur „antibarbarischen“ Distinktion (nach dem Motto: Die Masse meiden) haben, tendieren die jüngeren Milieus eher zu weniger (oder anders) bindungsintensiven Sozialformen, z. B. zu Szenenbildungen (vgl. Hitzler/Bucher/Niederbacher 2001).

Es empfiehlt sich also bereits auf der Stufe der Wahrnehmungsschulung zu lernen, nicht nur die Sinus-Milieus untereinander zu unterscheiden, sondern auch in sich selbst und dabei ihre jeweiligen sozialen Formierungen $z \mathfrak{u}$ beachten. Mit demselben Begriff (Sinus-Milieu) werden somit Milieulagerungen, Milieuzusammenhänge und/oder Milieueinheiten bezeichnet, also soziologisch durchaus unterscheidbare und hinsichtlich ihrer sozialen Verankerung, Bedeutung, Dynamik, Zukunftsfähigkeit und kommunikative Ansprechbarkeit recht unterschiedliche soziale Verbundenheiten.

\section{Literatur}

Barz, Heiner/Tippelt, Rudolf (2004): Weiterbildung und soziale Milieus in Deutschland. 2 Bände, Bielefeld.

Bohnsack, Ralf (1993): Rekonstruktive Sozialforschung. Opladen.

Ebertz, Michael N. (2006): Anschlüsse gesucht. Ergebnisse einer neuen Milieu-Studie zu den Katholiken in Deutschland. In: Herder Korrespondenz, Jg. 60, H. 4, S. 173177.

Hitzler, Ronald/Bucher, Thomas/Niederbacher, Arne (2001): Leben in Szenen. Formen jugendlicher Vergemeinschaftung heute. Opladen.

Mannheim, Karl (1964): Das Problem der Generationen. In: Ders.: Wissenssoziologie. Berlin, Neuwied, S. 509-565.

Mannheim, Karl (1980): Strukturen des Denkens. Frankfurt.

Müller-Schneider, Thomas (2003): Differenzierung des Milieugefüges? Eine zeitvergleichende Klassifikationsanalyse. In: Kölner Zeitschrift für Soziologie und Sozialpsychologie, Jg. 55, H. 4, S. 782-794.

Wippermann, Carsten/Magalhaes, Isabel (2005): Milieuhandbuch. Religiöse und kirchliche Orientierungen in der Sinus-Milieus 2005. Hg. von der MDG. München. 